\title{
ANEMIA EM PESSOAS DA CIDADE DE CÁCERES/MT E SUAS RELAÇÕES COM RENDA PER CAPITA
}

\author{
E.M.C. SANChES (1), M.D. de BARROS (2) \& P.R.B. de MELLO (3)
}

\begin{abstract}
RESUMO
Foram estudadas a anemia e suas relaçoes com a renda per capita em 1178 pessoas na cidade de Cáceres, Mato Crosso, Brasil nas faixas etárias de 6 a 14 anos (ambos os sexos), 15 a 45 anos do sexo feminino e 15 a 45 anos do sexo masculino.

Encontrou-se maior prevalência de pessoas anêmicas nas faixas de renda $<1 / 4$ do salário mínimo e $1 / 4-1 / 2$ do salário mínimo nas três faixas etárias estudadas.

Observou-se associação estatística entre a renda per capita e as médias de hemoglobina para as três faixas etárias.
\end{abstract}

UNITERMOS: Anemia; Faixa Etária; Renda per capita.

\section{INTRODUÇÃO}

A anemia nutricional é definida como uma condição na qual o conteúdo de hemoglobina do sangue é menor que o normal't. Resulta-se da deficiencia de um ou mais nutrientes essenciais, como ferro, Vitamina B-12, folato e outros microelementos como o zinco, manganês e o cobre.

A anemia devido à deficiência de ferro é o tipo mais comum da anemia nutricional, sendo de relevância não só nos países em desenvolvimento como naqueles altamente industrializados 1 .

A deficiência de ferro se processa no organismo de maneira graduada e progressiva, resultando tardiamente no aparecimento da anemia. Os principais fatores implicados neste processo são: o crescimento rápido, o aporte de ferro alimentar insuficiente, as perdas sanguíneas conseqüentes às parasitoses intestinais e às perdas menstruais .

Devido às suas conseqüências, a anemia nutricional representa um problema fundamental em saúde pública por ser prejudicial ao crescimento, facilitar a instalação de processos infecciosos, diminuir o rendimento do aprendizado e a produtividade no trabalho ${ }^{6}$.

Estudos realizados anteriormente em 1983, na cidade de Cáceres, revelaram uma alta prevalência de anemia. O presente estudo teve como objetivos:

1) verificar os indices de anemia por faixa etária;

2) verificar a freqüência de anemia nas faixas etárias conforme a renda per capita.

(1) Farmacêutica-Bioquímica -- Bolsista supervisora do grupo hematológico

(2) Doutora em Pediatria UFMT - Coordenadora do Projeto;POLONOROESTE

(3) Mestre em Pediatria UFMT.

Endereço para correspondência: Universidade Federal de Mato Grosso - Av. Fernando Corrêa, s/no - 78100 Cuiabá, Mato Grosso, Brasil 
SANC HLS. L.M1. . BARROS, M.D. de \& MEI.I.O, P.R.B. de - Anemia em pessoas da cidade de Cáceres/MT e suas relaçōes com renda per capita. Rè. Inst. Med. trop. São Paulo, 30(3):165-168, 1988

\section{MATERIAL E MÉTODOS}

\section{Região}

A região de estudo compreendeu a área de influência do Projeto Polonoroeste em Mato Grosso, tendo como cidade representativa Cáceres, inserida no Pantanal Matogrossense. É uma região caracterizada por baixa altitude, inferior a $300 \mathrm{~m}$.

\section{Amostras}

A população de estudo foi delineada a partir da amostra do grupo de Inquérito Epidemiológico por Entrevistas Domiciliares do Polonoroeste/MT, que seguiu o estabelecido na Pesquisa Nacional por Amostra de Domicílios (PNAD). Foram convidadas a participar do estudo pessoas na faixa etária de 6 a 45 anos de idade.

As pessoas for am estratificadas por renda, idade e sexo. As frações salariais foram estabelecidas através da renda per capita assim divididas:

1) menor ou igual a 1/4 de salário mínimo;

2) mais que $1 / 4$ e menor ou igual a $1 / 2$ de salário mínimo;

3) maior que $1 / 2$ salário mínimo e menor ou igual a 1 salário mínimo;

4) maior que 1 salário mínimo.

As faixas etárias consideradas foram:

a) 6 a 14 anos (para ambos os sexos)

b) 15 a 45 anos (sexo masculino)

c) 15 a 45 anos (sexo feminino)

\section{Exames Laboratoriais}

Foram colhidos $1,5 \mathrm{ml}$ de sangue por pun cào venosa periférica em frascos contendo E.D.T.A. para as dosagens de hemoglobina.

O material foi remetido por via terrestre ao Laboratório de Análises Clínicas do Polonoroeste na Universidade Federal de Mato Cirosso, distante $240 \mathrm{~km}$ do local da coleta. A dosagem de hemoglobina foi realizada com sangue total em 1.178 pessoas, através de um Hemoglobinômetro da Coulter, 6 horas após a coleta. Como critério de diagnóstico de anemia cumbiderou-se on valores adolados pela onss:
6 a 14 anos (ambos os sexos) $=12 \mathrm{~g} / \mathrm{dl}$ 15 a 45 anos (masculino) $=13 \mathrm{~g} / \mathrm{dl}$ 15 a 45 anos (feminino) $=12 \mathrm{~g} / \mathrm{dl}$.

\section{Estatística}

Para o estudo estatístico utilizou-se a análise de variância e o teste de Tuchey para a verifícação de possíveis diferenças entre as concentrações de hemoglobina, faixas etárias e níveis de renda. O nivel de significância estipulado foi $=0.01$.

\section{RESULTADOS}

Utilizando-se o critério de nivel de hemoglobina, verificou-se que das 1.178 pessoas examinadas, ocorreu uma porcentagem de anemia elevada na faixa etária de 6 a 14 anos e decrescendo para a faixa de 15 a 45 anos, tanto para o sexo feminino como para o masculino (tabela 1).

Quando estratificamos a amostra por renda per capita, observamos também a percentagem de anemia mais alta na faixa etária de 6 a 14 anos (ambos os sexos), sendo esta maior na faixa menor que $1 / 2$ salário mínimo e decrescente nas faixas acima de $1 / 2$ salário mínimo (tabela 2). Ocorreu o mesmo para as faixas etárias de 15 a 45 anos do sexo masculino e feminino (tabelas 3 e 4 ).

Existiu diferença significativa entre os valores médios de hemoglobina de acordo com a faixa de renda per capita e esses valores foram maiores nas faixas etárias de 6 a 14 anos, em que o valor médio de hemoglobina foi maior na faixa de renda $<1 / 4$ salário mínimo do que $1 / 4$ a $1 / 2$ salário mínimo.

A análise de variância ( $\mathrm{F}$ ) mostrou que as diversas faixas de renda per capita atuaram como uma fonte de variação estatisticamente significativa, no comportamento das médias de hemoglobina na amostra estudada $(p<0.01)$. O teste de Tuchey evidenciou que existiu diferenfas significativas das médias de hemoglobina nas rendas per capita estudadas.

\section{DISCUSSĀO}

Os resultados apresentados vem reforçar a 
SANCHES, E.M.C.; BARROS, M.D. de \& MELLO, P.R.B. de - Anemia em pessoas da cidade de Cáceres/MT e suas relaçסes com renda per capita. Rev. Inst. Med, trop. São Paulo, 30(3):165-168, 1988.

importância das anemias entre as endemias carenciais de maior prevalência em nosso meio.

\section{TABELA}

Valores de hemoglobina (média \pm desvio padrão) ( $\mathrm{g} / \mathrm{dl}$ ) e percentual de anemia em pessoas por faixa etária e sexo na cidade de Cáceres/MT - 1985.

\begin{tabular}{lccc}
\hline Idade & $\mathrm{n}$ & Hgb & \% Anemia \\
\hline $\begin{array}{l}\text { S-14 anos } \\
\text { (Ambos os sexos) }\end{array}$ & 547 & $\begin{array}{c}12,28 \\
\pm 1,15)\end{array}$ & 36,12 \\
$\begin{array}{l}15-45 \text { anos } \\
\text { (Masculino) }\end{array}$ & 263 & $\begin{array}{c}14,13 \\
( \pm 1,41)\end{array}$ & 17,11 \\
$\begin{array}{l}15-45 \text { anos } \\
\text { (Feminino) }\end{array}$ & 378 & $\begin{array}{c}12,69 \\
( \pm 1,14)\end{array}$ & 20,89 \\
\hline
\end{tabular}

TABELA 2

Valores de hemoglobina (média \pm desvio padrão) $(\mathrm{g} / \mathrm{dl})$ e percentual de anemia em pessoas de 6 a 14 anos (ambos os sexos) e renda per capita na cidade de Cáceres/MT, 1985.

\begin{tabular}{lccc}
\hline $\begin{array}{c}\text { Salário Mínimo } \\
\text { (S.M) }\end{array}$ & $\mathrm{n}$ & $\mathrm{Hgb}$ & \% Anemia \\
\hline$<1 / 4$ S.M & 184 & $\begin{array}{c}12,11^{* *} \\
( \pm 1,26)\end{array}$ & 40,76 \\
& 161 & $\begin{array}{c}12,05^{* *} \\
( \pm 1,13)\end{array}$ & 47,20 \\
$1 / 4$ S.M - 1/2 S.M & 124 & $\begin{array}{c}12,48^{* *} \\
( \pm 1,14)\end{array}$ & 27,42 \\
$1 / 2-1$ S.M. & & $\begin{array}{c}12,92 * * \\
( \pm 1,17)\end{array}$ & 13,24 \\
\hline 1 S.M & 68 & & \\
\hline
\end{tabular}

$F(3,533)=11,8(p<0,01)$

Tuchey** d.m.s. $=0.05$

No Brasil, nos últimos anos, algumas pesquisas de campo e revisões bibliográficas tem ressaltado a elevada prevalência das anemias, embora sejam bastante discordantes os resultados obtidos em diferentes áreas, segundo diferentes autoress. Podemos observar que a maioria restringe-se as laixas chálias mais vulneraveis a tal afecção como os pré-escolares e as gestantes. Sendo assim, o indice é estritamente elevado como $510 \%$ registrado por SAlLANO et al.s e $34 \%$ por SIGULEM et al.t
TABELA 3

Valores de hemoglobina (média \pm desvio padrão $(\mathrm{g} / \mathrm{dl}$ ) e percentual de anemia em pessoas entre 15 a 45 anos do sexo feminino e renda per capita na cidade de Cáceres/MT, 1985.

\begin{tabular}{lccc}
\hline $\begin{array}{c}\text { Salário Mínimo } \\
\text { (S.M.) }\end{array}$ & $\mathrm{n}$ & $\mathrm{Hgb}$ & \% Anemia \\
\hline$<1 / 4$ S.M & 93 & $\begin{array}{c}12,38^{* *} \\
( \pm 1,26)\end{array}$ & 32,26 \\
1/4 S.M - 1/2 S.M & 99 & $\begin{array}{c}12,59^{* *} \\
( \pm 0,98)\end{array}$ & 25,25 \\
$1 / 2-1$ S.M & 108 & $\begin{array}{c}12,80^{* *} \\
( \pm 1,15)\end{array}$ & 17,57 \\
> I S.M & 78 & $\begin{array}{c}13,06^{* *} \\
( \pm 1,17)\end{array}$ & 6,41 \\
& & &
\end{tabular}

$F(3,374)=15,5(p<0,01)$

Tuchey**d.m.s. $=0.06$

TABELA 4

Valores de hemoglobina (média \pm desvio padrão) $(\mathrm{g} / \mathrm{d} 1)$ e percentual de anemia em pessoas entre 15 a 45 anos do sexo masculino e renda per capita na cidade de Cáceres/MT, 1985.

\begin{tabular}{lccc}
\hline $\begin{array}{c}\text { Salário Mínimo } \\
\text { (S.M.) }\end{array}$ & $\mathrm{n}$ & $\mathrm{Hgb}$ & \% Anemia \\
\hline$<1 / 4$ S.M & 60 & $\begin{array}{c}13,80^{* *} \\
( \pm 1,73)\end{array}$ & 25,00 \\
1/4 S.M - 1/2 S.M & 81 & $\begin{array}{c}13,91^{* *} \\
( \pm 1,26)\end{array}$ & 18,52 \\
$1 / 2-1$ S.M. & 75 & $\begin{array}{c}14,31^{* *} \\
( \pm 1,20)\end{array}$ & 14,67 \\
>1 S.M & 47 & $\begin{array}{c}14,62 \\
( \pm 1,36)\end{array}$ & 8,51 \\
\hline
\end{tabular}

$\mathrm{F}(3,259)=6,18(\mathrm{p}<0,01)$

Tuchey** d.m.s. $=0.08$

VALLADA et al. ${ }^{8}$ estudaram pessoas entre 1 a 80 anos de idade na região de Sorocaba-SP. Nesta região os índices de anemia diferiram daqueles obtidos por nós. Estes autores encontraram um índice de $23,7 \%$ para a faixa etária de 6 a 14 anos e $25,2 \%$ e $18,5 \%$ para a faixa de 15 a 45 anos referente ao sexo masculino e feminino, respectivamente. No presente estudo, para estas mesmas faixas, detectamos índices de 36,2\%, $20,2 \%$ e $28,2 \%$. Indices diferentes também foram relatados por MARTINS et al. ${ }^{3}$ que estu- 


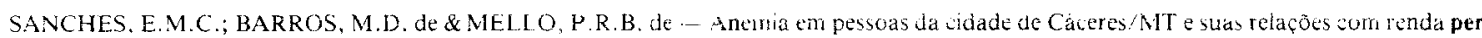
capita. Rev. Inst. Med. 1rop. São Paulo, 30(3):165-168, 1988

daram padrões hematologicos em 400 pessods de todas as faixas etárias na zona da Mara.PE. Na faixa de 6 a 14 anos o indice foi de $4,4 \%$ e de $33,3 \%$ no grupo masculino acima de 15 anos. Dessa forma, podemos verificar que a anemia não é um problema que atinge uma única faixa etária, mas acomete igualmente todos os grandes agrupamentos etários, sejam crianças ou adultos de ambos os sexos, com o que corroboram os nossos resultados.

Por fïm, questiona-se a possível vinculação entre a renda per capita e a prevalência de aneniia, partindo-se da observação de que, como muitas vezes ocorre, as carências nutricionais acham se associadas às condiçoes sócioeconônicas dos hospedeiros'. Tal suposição tora corroborada pelos resultados encontrados por SALZANO et als, SIGULEM et al." e por nós neste estudo, estabelecendo-se que a renda familia iem efeito marcante na pcorrencia de anemias, sendo que existe uma tendencia à li. minuiçào da frequência de anemia à medida que esia renda cresce.

\section{SUMMARY}

\section{Anemia in inhabitants of Cáceres/MT and its correlation with income per capita}

A sucil was made about anemia and its relationship with the income/per capita among 11:8 individuals beween the age of 6 -it year old (of both sex), 15 to 45 years old temale, 15 to 45 years old male.

It showed higher prevalence of anemic individuals belonging to the income ranges of $1 / 4$ minimum salary and $1 / 4-1 / 2$ minimum salary within the three ranges of ages studied.
It was observed a statistical relationship between the incorne/per capita and the average rates of hemoglobin for the three ranges of age.

\section{AGRADECIMENTOS}

Agradecemos ao Prof. PAULO SABROZA pelas sugestōes prestadas no desenvolvimento deste trabalho.

\section{REFERENCIAS BIBLIOGRAFICAS}

1. BRANDELISE, S.R. \& MATSUDA, E. - Anemias carenciais. In: NÓBREGA, F. ... Desnutrição intrauterina e pós-natal. São Paulo, Panamed, 1981, p. 395411

2. CANTO DE CETINA, T.E.; CARDENAS, S.; ORTIZ, M.E.; POLANCO, L.; VER.A, L.; PINÄ-CASTRO, R. \& CUPUL, $G$ - Valores de hemoglobina $y$ hierro serico en mujeres de ciase socioeconomica baja lucatan, Mexico. Bol. Of́ic. sanit panamer., 98: 464472,1985

3. MARTINS, G.C.; SALIANO. A.C.; BATISIA, M.\& VARELA, R.M. - Padròes hematologicos em grupos populacionais da zena da Mata de P'ernambuco. Rev. bras. Pesq. méd. Biol., 4: 399-403, 1971.

4. NUTRITIONAL ANAEMIAS. Wid. Hith. Org. tectin. Rep. Ser., (405), 1968.

5. SALZANO, A.C.; TORRES, M.A.A.; BATISTA F1. LHO, M. \& ROMANI, S. de A.M. -- Anemia em crianças de dois serviços de saúde de Recife (PE), Brasil. Rev. Saúde públ. (S. Paulo), 19: 499-507, 1985.

6. SIGULEM, D.; TUDISCO, E.S.; GOLDENBERG, P.; ATHAIDE, M.M.M. \& VAISMAN, E. - Anemia ferropriva em crianças do município de São Paulo. Rev. Saúde públ. (S. Paulo), 12: 168-178, 1978.

7. SZARFARC, S.C. - Diagnóstico de deficiência de ferro na infância. Rev. Saúde púbı. (S. Paulo), 19: 278-284, 1985.

8. VALLADA, E.P. et al. - Distribuição dos niveis de hemoglobina em crianças, adultos e idosos na população da região de Sorocaba. (Nimeogratado) 